\title{
ÚNG DỤNG WAVELET ĐỂ CHIẾT XUẤT ĐIỂM ĐẶC TRƯNG PHỤC VỤ ĐỒNG ĐĂNG KÝ ẢNH SAR
}

\author{
TRÀN THANH HÀ \\ Trường Đại học Mỏ - Địa chất
}

\section{Tóm tắt:}

Việc lựa chọn tập hợp các điểm khớp là một trong những bước quan trọng của quá trình đồng đăng kí ảnh SAR. Những phần mềm xử lý ảnh thương mại hiện nay cho phép việc lựa chọn các điểm khớp dựa vào các điểm mắt lưới. Điều đó có nghĩa là khoảng các giữa các điểm khớp phụ thuộc vào việc thiết lập khoảng cách mắt lưới. Như vậy, tính tương quan của các điểm khớp sẽ bị giảm do đó sẽ ảnh hưởng đến độ chính xác của quá trình đồng đăng ký ảnh. Tuy nhiên, độ chính xác này có thể được nâng cao bằng cách tìm ra những điểm đặc trưng trên ảnh để sử dụng làm điểm khớp, và độ chính xác khi xây dựng DSM sẽ đáng tin cậy hơn. Bài báo trình bày một trong những phương pháp có thể được sử dụng để tìm ra điểm đặc trung, phương pháp wavelet.

\section{Giới thiệu}

Khi đồng đăng ký ảnh dựa vào các điểm đặc trưng thì sự phân bố của các điểm đặc trưng trên ảnh là một trong các yếu tố quan trọng ảnh hưởng tới độ chính xác của đồng đăng ký ảnh. Nếu điểm đặc trưng được phân bố đều trên toàn ảnh thì việc đồng đăng ký sẽ đáng tin cậy hơn so với các điểm phân bố không đều. Quá trình khớp điểm ảnh đặc trưng bao gồm hai bước: chiết xuất điểm ảnh đặc trưng và khớp điểm ảnh đặc trưng. Hiện nay, một số phương pháp được sử dụng để chiết xuất các điểm đặc trưng từ ảnh. Một trong những phương pháp đó là phương pháp Interest Operators (IO) (Urban, 2003). Phương pháp này sử dụng giá trị gradient cực đại để chiết xuất các điểm đặc trưng. Tuy nhiên việc tính giá trị gradient cho mỗi điểm ảnh từ ảnh gốc tạo nên khối lượng tính toán vô cùng lớn. Phương pháp wavelet cũng được sử dụng để chiết xuất các điểm đặc trưng từ ảnh (Moigne et al., 2002). Phương pháp này dựa trên giá trị wavelet gradient lớn nhất. So sánh Interest Operators (IO) với wavelet, thì giá trị gradient không tính cho ảnh gốc nhu $\mathrm{IO}$ mà chỉ được tính cho các ảnh ở các tầng phân tích wavelet, do đó giảm được khối lượng tính toán. Đặc biệt wavelet còn hỗ trợ cho phân tích đa phân giải ảnh. Phân tích wavelet cung cấp tất cả các đặc trưng của ảnh mà không làm mất đi thông tin quan trọng của dữ liệu ban đầu ngay cả ở độ phân giải thấp. Ngoài ra các tính chất của hệ số wavelet sử dụng để lọc tần số thấp và tần số cao còn được sử dụng trong việc chiết xuất đặc trưng của ảnh (Louis et al, 1997), và nó có thể sử dụng để tìm điểm đặc trưng.

Tóm lại, việc sử dụng wavelet cho phân tích đa phân giải để tìm điểm đặc trưng phục vụ cho quá trình đồng đăng ký là rất hợp lý vì:

- Các điểm đặc trưng phải là các điểm như ngã ba đường, góc nhà, các giao lộ, các địa vật độc lập ... Tuy nhiên những điểm đặc trưng này không phải lúc nào cũng tìm thấy trên ảnh SAR. Vì vậy, ý nghĩa của điểm đặc trưng rất quan trọng. Trong nghiên cứu

Ngày nhận bài: 28/8/2017, ngày chuyển phản biện: 05/9/2017, ngày chấp nhận phản biện: 13/9/2017, ngày chấp nhận đăng: 20/9/2017 
này điểm đặc trưng được định nghĩa trong miền wavelet.

- Trong phương pháp phân tích đa phân giải bằng wavelet thì dữ liệu gốc không bị mất đi mà các đặc trưng của tín hiệu lại được tìm thấy ngay cả ở độ phân giải thấp.

- Phân tích wavelet đa phân giải sẽ làm nổi bật đặc trưng của hình ảnh ở độ phân giải thấp, do đó nó loại bỏ được một số đặc trưng không rõ ràng ở độ phân giải cao.

- So với các phương pháp khác, thuật toán này đạt độ chính xác cao hơn với tốc độ tính toán nhanh và ở tất cả các độ phân giải.

\section{Cơ sở của phương pháp}

Phân tích trên ảnh có thể được thực hiện bằng phân tích đa phân giải wavelet 2-D

Trong không gian 2-D:

nếu hàm $\Psi(x, y) \in L^{2}\left(R^{2}\right)$

thỏa mãn: $\iint_{R^{2}} \Psi(x, y) d x d y=0$

Nó được gọi là wavelet hai chiều cơ bản "wavelet mẹ". Wavelet 2-D liên tục biến đổi theo hàm $f(x, y) \in L^{2}\left(R^{2}\right)$ được cho bởi hàm tích phân chập

$$
w_{a}[f(x, y)]=\iint_{R^{2}} f(x, y) \Psi_{a, b}(x, y) d x d y=f * \Psi_{a}(x, y)
$$

Biến đổi wavelet có thể được coi như là bộ lọc của băng tần, có thể thực hiện ở các loại tỷ lệ và độ phân giải khác nhau. Nếu chúng ta dùng cửa sổ lớn để nhìn vào dữ liệu thì chung ta sẽ thấy đặc trưng tổng thể, tương tự, nếu chúng ta dùng cửa sổ nhỏ để nhìn vào dữ liệu thì chúng ta sẽ thấy các đặc trưng chi tiết. Đối với từng lớp nhỏ của wavelet, tỷ lệ a có thể được lấy mẫu liên tục $a=2^{i}$ với $j \in Z$, mà không thay đổi các thuộc tính tổng thể của phép biến đổi. Các phép biến đổi tương ứng với giá trị nhân đôi của a được gọi là biến đổi wavelet rời rạc (DWT), $\quad w_{2^{j}}[f[x, y]]=f * \Psi_{2^{j}}(x, y)$

Do đó, hình ảnh có thể được coi là một chức năng $f(x, y)$ và biến đổi wavelet rời rạc có thể thực hiện theo mô tả theo công thức 2.3.

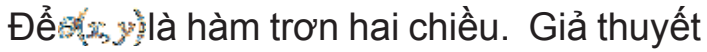
rằng $\sin _{3}, y_{y}^{2}$ được phân tích thành hai thành phần dọc theo hướng $\mathrm{x}$ và $\mathrm{y}$ là:

$$
\begin{gathered}
\Psi^{1}(x, y)=\frac{\partial \theta(x, y)}{\partial x} \\
\Psi^{2}(x, y)=\frac{\partial \theta(x, y)}{\partial y}
\end{gathered}
$$

Các chức năng này có thể được sử dụng như wavelet. Ở tỷ lệ $a=2 j$, Biến đổi wavelet 2-D của ảnh $f(x, y)$ có thể được phân tích thành hai hướng độc lập.

$$
\begin{aligned}
& w_{2^{j}}^{1}[f(x, y)]=f * \Psi_{2^{j}}^{1}(x, y) \\
& w_{2^{j}}^{2}[f(x, y)]=f * \Psi_{2^{j}}^{2}(x, y)
\end{aligned}
$$

Về cơ bản, hai thành phần này tỷ lệ thuận với tọa độ của vector hướng dốc của $f(x, y)$, làm trơn bằng ${ }^{\prime}\left(s_{2}\right)$ ở tỷ lệ $2^{j}$ theo hai hướng $x$ và $y$.

Ở tỷ lệ $2 \mathrm{j}$, môdul vector hướng dốc của $\mathrm{f}(\mathrm{x}, \mathrm{y})$ có thể được tính như sau:

$$
M_{2^{j}}[f(x, y)]=\sqrt{\mid w_{2^{j}}^{1}\left[\left.f(x, y)\right|^{2}+\mid w_{2^{j}}^{2}\left[\left.f(x, y)\right|^{2}\right.\right.}
$$

Ở mỗi tỷ lệ $2^{\mathrm{j}}$, biến đổi wavelet cực đại được định nghĩa là các điểm $(x, y)$ mà tại đó môdul $M_{2^{j}}[f(x, y)]$ là cực đại địa phương dọc theo hướng dốc.

\section{Phương pháp chiết xuất các điểm đặc trưng bằng wavelet}

\subsection{Phân tích tín hiệu SAR bằng wavelet}

Quá trình chiết tách các điểm đặc trưng trên ảnh $S A R$ được bắt đầu bằng việc phân tích đa phân giải ảnh SAR. Phép biến đổi wavelet rời rạc được sử dụng để phân tích đa phân giải ảnh SAR (hình 1). Trong phần thực nghiệm tác giả sử dụng ảnh Sentinel - 1 A được Cơ quan hàng không vũ trụ Châu Âu phóng thành công lên quỹ đạo năm 2014 với chu kỳ là 12 ngày. Đây là tư liệu viễn thám mới và có độ phân giải không gian $10 \mathrm{~m}$, cung cấp dữ liệu SAR band $\mathrm{C}$ bước 
sóng $5,66 \mathrm{~cm}$ với chế độ chụp là IW. Các phép lọc được tiến hành với nhiều tầng (level) khác nhau và để khối lượng tính toán không tăng, khi qua mỗi bộ lọc, tín hiệu được lấy mẫu xuống 2. (xem hình 1)

Mỗi thành phần của ảnh được phân tích ở mỗi tầng phân tích nêu trên được xem như là một kênh ảnh. Như vậy, ở mỗi tầng phân tích, ảnh được phân tích thành 4 ảnh nhỏ hơn. Hình 2 mô tả cấu trúc phân tích hình kim tự tháp của phương pháp wavelet. Ảnh SAR được phân tích bằng wavelet để chuẩn bị cho bước chiết tách điểm ảnh đặc trưng tiếp theo. (xem hình 2)

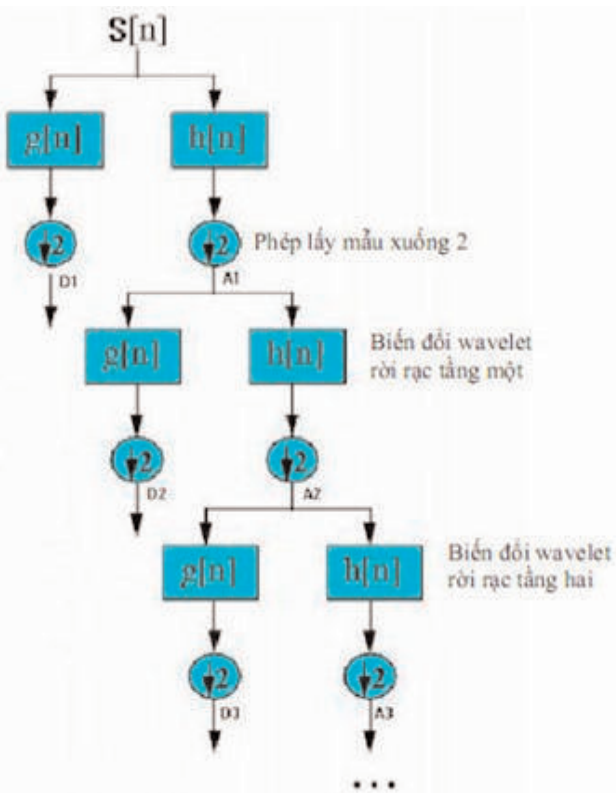

Hình 1: Sơ đồ phân tích ảnh bằng wavelet

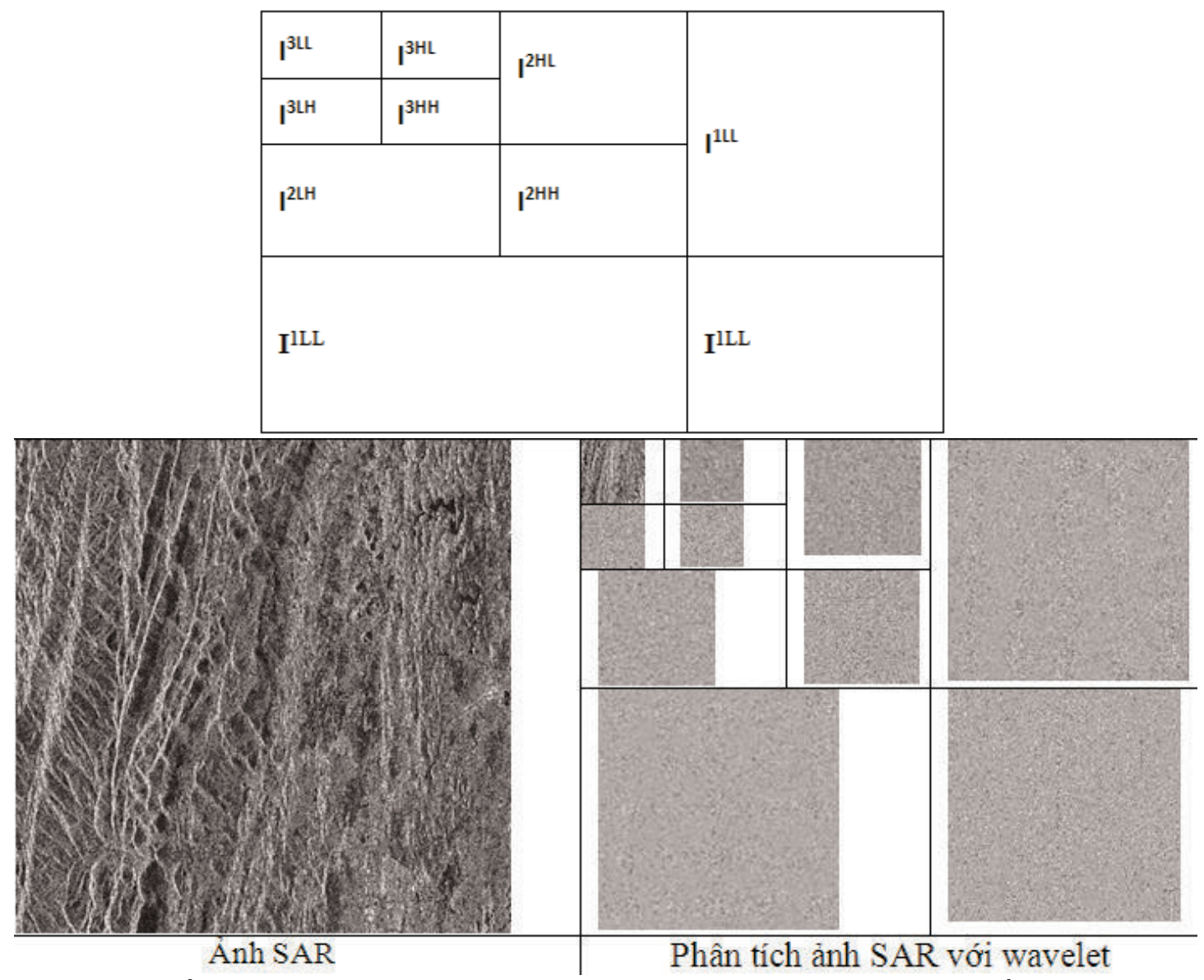

Hình 2: Cấu trúc hình kim tự tháp của phương pháp phân tích ảnh bằng wavelet 


\subsection{Chiết xuất điểm đặc trưng}

Khi ảnh SAR đã được phân tích thành các mức khác nhau, thì bước tiếp theo là xác định các điểm đặc trưng trên hai ảnh ở từng lớp khác nhau. Trong phần nghiên cứu này tác giả sử dụng môdul cực đại của biến đổi wavelet để phát hiện sự thay đổi sắc nét của các điểm trên ảnh. Mức $L H$ và $H L$ trong biến đổi wavelet được sử dụng để ước lượng hình ảnh dốc.

Ở mức thấp nhất, tham số $\lambda_{2}$ là ngưỡng để phát hiện ra điểm đặc trưng. Tại mỗi điểm chuyển đổi wavelet được xác định theo công thức (2.6). Những điểm nằm trong ngưỡng $\lambda_{2}$ thì được chọn là điểm đặc trưng.

Số điểm đặc trưng được chọn là 36,49 , 64, 81 điểm khác nhau để kiểm chứng cho đánh giá độ chính xác. Đối với phương pháp lưới grid điểm đặc trưng được lựa chọn với khoảng cách giữa các điểm trong lưới là 200 theo hàng và 30 theo cột, còn đối với phương pháp wavelet thì các điểm được chọn dựa trên đặc trưng của ảnh. Để so sánh sự khác nhau trong hai phương pháp này, thì các điểm đều được chọn trên ảnh SAR thô (SAR raw). Và đánh giá độ chính xác của hai phương pháp dựa vào tổng độ lệch pha (SPD - sum of the diference) với công thức tính như sau:

$$
S P D=\sum_{x=0}^{p-1} \sum_{y=0}^{q-1} S P D_{\text {local }}(x, y)
$$

Trong đó $p \times q$ là kích thước của ảnh, $\operatorname{SPD}_{\text {local }}(x, y)$ là độ lệch pha của pixel có vị trí hàng $x$, cột $y$. (xem bảng 1 )

\section{Kết luận}

Các kết quả cho thấy đối với phương pháp truyền thống sử dụng mắt lưới grid để xác định các điểm đặc trưng phục vụ cho đồng đăng ký ảnh cho kết quả cũng tương đối cao. Tuy nhiên phương pháp này cũng có những ưu nhược điểm riêng. Ưu điểm là các điểm được phân bố để trên toàn ảnh còn nhược điểm là một số các điểm được chọn có độ tương quan không cao, vì vậy để nâng cao độ chính xác của quá trình đồng đăng ký ảnh là rất khó khăn. Dựa vào tổng độ lệch pha (SPD) thì phương pháp wavelet cho độ chính xác cao hơn phương pháp mắt lưới grid, điều này chứng tỏ các điểm được chọn có độ tương quan tương đối cao và cũng khẳng định rằng nếu sử dụng phương pháp wavelet để chiết xuất các điểm đặc trưng thì sẽ nâng cao được độ chính xác của quá trình đồng đăng ký ảnh. Tuy nhiên, điều này chỉ đúng với trường hợp các điểm đặc trưng được phân bố đều trên ảnh. Trong các trường hợp mà các điểm đặc trưng phân bố không đều trên ảnh, nếu chỉ sử dụng điểm đặc trưng này thì có thể dẫn tới việc đồng đăng ký ảnh có độ chính xác thấp. Do đó, việc sử dụng cả điểm đặc trưng và điểm mắt lưới có thể khắc phục vấn đề này. $\mathrm{O}$

\section{Bảng 1: Kết quả so sánh độ chính xác của hai phương pháp}

\begin{tabular}{|c|c|c|}
\hline \multirow{2}{*}{ Số điểm đặc trung } & Kết quả điểm đặc trưng theo lưới Grid & Kết quả điểm đặc trưng theo wavelet \\
\cline { 2 - 3 } & SPD (radian) & SPD (radian) \\
\hline 36 & 42,956 & 41,490 \\
\hline 49 & 45,062 & 41,183 \\
\hline 64 & 41,823 & 40,211 \\
\hline 81 & 43,887 & 40,707 \\
\hline
\end{tabular}



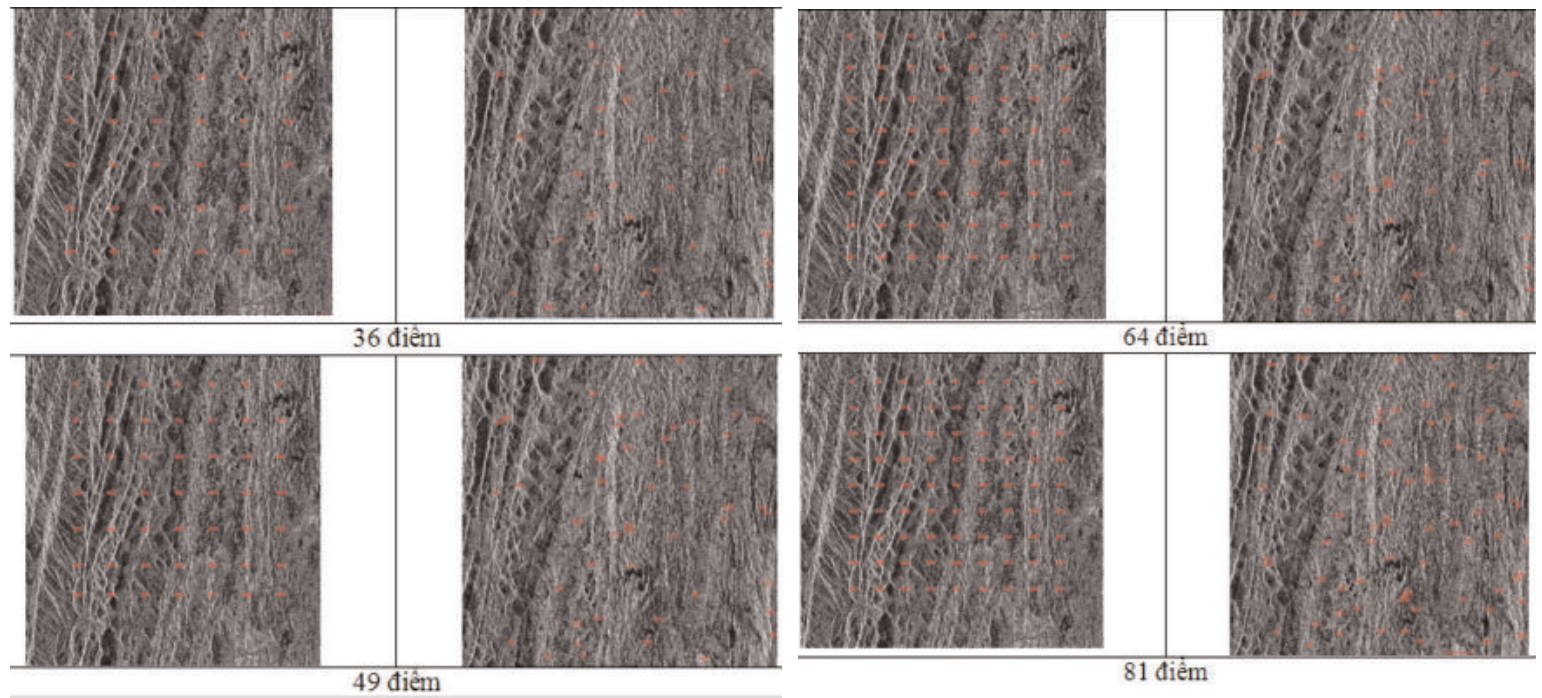

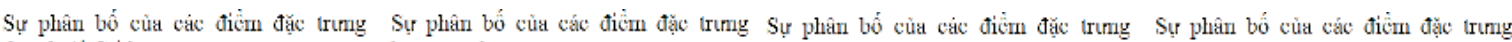
theo lưới Grid theo wavelet theo lưới Grid theo wavelet

Hình 3: Sự phân bố của các điểm đặc trưng trên ảnh SAR

\section{Tài liệu tham khảo}

[1]. F. Yin and M. Lin, 1996 "Wavelet Transform with Application on Radar Signal Synthesis," in Proc. IEEE CIE International Conference of Radar, pp. 293- 296.

[2]. Louis, A.K, Maass,A. Rieder, 1997. Wavelets: Theory and Applications, 342p.
[3]. Moigne, J.L., Campbell, W.J. and Cromp, R. F, 2002. An Automated Parallel Image Registration Technique Based on the Correlation of Wavelet Features, IEEE International Geoscience and Remote Sensing Symposium, 40(8):1849-1864. O

\section{Summary}

\section{Applying wavelet techniques in extracting feature points for co-registration SAR} images

\section{Tran Thanh $\mathrm{Ha}$}

\section{Hanoi University of Mining and Geology}

The process of coregistration SAR images involves a step of choosing a set of tie points. Several current commercial image processing software allows to select nodes of the grid as tie points. Thus, the interval between the tie points depends on the pre-set distance (or interval) of the grid nodes. This may cause to a low cross-relationship of some tie points, then the accuracy of the process of co-registration is affected. However, the accuracy of this process can be improved if feature points are identified on SAR images, and used. As results, the accuracy of DSM generated by SAR image is more reliable. This article discusses about one of techniques can be applied in extracting feature points on image, the wavelet technique. $\mathrm{O}$ 